\title{
Finite-Time Composite Position Control for a Disturbed Pneumatic Servo System
}

\author{
Xiaojun Wang, Jiankun Sun, and Guipu Li \\ Key Laboratory of Measurement and Control of Complex Systems of Engineering, Ministry of Education, School of Automation, \\ Southeast University, Nanjing 210096, China
}

Correspondence should be addressed to Xiaojun Wang; 101011042@seu.edu.cn

Received 5 May 2016; Accepted 19 October 2016

Academic Editor: R. Aguilar-López

Copyright (C) 2016 Xiaojun Wang et al. This is an open access article distributed under the Creative Commons Attribution License, which permits unrestricted use, distribution, and reproduction in any medium, provided the original work is properly cited.

This paper investigates the finite-time position tracking control problem of pneumatic servo systems subject to hard nonlinearities and various disturbances. A finite-time disturbance observer is firstly designed, which guarantees that the disturbances can be accurately estimated in a finite time. Then, by combining disturbances compensation and state feedback controller together, a nonsmooth composite controller is developed based on sliding mode control approach and homogeneous theory. It is proved that the tracking errors under the proposed composite control approach can be stabilized to zero in finite time. Moreover, compared with pure state feedback control, the proposed composite control scheme offers a faster convergence rate and a better disturbance rejection property. Finally, numerical simulations illustrate the effectiveness of the proposed control scheme.

\section{Introduction}

Pneumatic servo systems play an important role and are widely used in modern industry (see pneumatic muscle systems [1, 2], pneumatic brake systems [3], pneumatic manipulators $[4,5]$, ball-plate pneumatic systems [6], etc.), since they have lots of advantages, such as energy saving, high powerto-weight ratio, low cost, simple structure and operation, and ease of maintenance $[7,8]$. A lot of industry applications require high-precision position control of pneumatic servo systems [9-12]. Unfortunately, there exist various disturbances and hard nonlinearities due to the compressibility of gas, nonlinearity of servo valve, and so forth in pneumatic servo dynamics systems $[8,9]$, which brings a challenge for position control design of pneumatic servo systems. Consequently, designing a controller to deal with hard nonlinearities and disturbances is very important for improving the tracking performance of the pneumatic servo systems.

Recently, in order to attenuate the undesirable influence caused by system parameter uncertainties and external disturbances, lots of robust control approaches have been developed and widely applied in practical systems, such as sliding mode control $[9,10,12]$, adaptive control $[13,14]$, disturbance observer based control $[1,2,15,16]$, and active disturbance rejective control [11]. Even though the aforementioned methods can attenuate the parameter uncertainties and external disturbances. However, their convergence rates are at best exponential, since most of the closed-loop pneumatic servo systems under the aforementioned control algorithms are Lipschitz continuous. As an alternative of smooth control, nonsmooth control is being more and more popular due to its many of advantages, such as faster convergence rates and better disturbance rejection properties [17, 18].

Nonsmooth control is an efficient method to achieve finite-time convergence; that is, the state of the closed-loop system under the nonsmooth control converges to zero in a finite time. Different from asymptotically stable systems, nonsmooth control systems are a kind of non-Lipschitz continuous ones, which leads to the difficulties in analysis and synthesis of nonsmooth control problems. Nonsmooth control has been widely investigated from the aspects of both theory and application, including results on finite-time stability analysis tools [18-20], lower-order systems [21, 22], cascaded system [23], and individual systems [24, 25]. In order to improve the closed-loop system performance, disturbance observer based control schemes composed of disturbance observer design and nominal feedback controller design have been proposed in [26-29]. Compared with pure feedback control methods, 
disturbance observer based control has several superiorities, such as faster rejection of disturbances and recovery of the nominal performances. In particular, the finite-time disturbance observer proposed in [30] can accurately estimate the disturbance in a finite time. Therefore, the undesirable influence caused by disturbances and uncertainties can be canceled in a finite time by disturbance compensation. However, to the best of our knowledge, there are no published results on finite-time disturbance observer based composite control design for pneumatic servo systems by using integral sliding mode control and homogeneous theory.

This paper studies the finite-time position control problem of a pneumatic servo system via integral sliding mode control approach and homogeneous theory [31]. Firstly, a finite-time disturbance observer is designed to estimate the disturbance. Then, by using homogeneous theory and integral sliding mode control approach, a composite finite-time controller combining disturbance compensation and state feedback is designed, which makes tracking errors globally converge to zero in a finite time. Finally, the effectiveness of the proposed control scheme is verified through numerical simulations. The main contributions of this paper are twofold. First and foremost, a finite-time disturbance observer is presented to estimate the disturbance in a finite time, such that the undesirable influence caused by the disturbance can be removed in a finite-time by using disturbance compensation. Second, compared with the smooth control schemes, the proposed finite-time composite control scheme belongs to nonsmooth control approach, which offers a faster convergence rate and a better disturbance rejection performance for the closed-loop tracking error system.

The remaining parts of this paper are organized as follows. Section 2 reviews some preliminary knowledge about some relevant basic concepts and lemmas. The pneumatic servo system model is constructed in detail in Section 3. The proposed composite controller and stability analysis are presented in Section 4. In Section 5, numerical simulations on the comparisons between the proposed composite controller and the pure state feedback controller are presented under the different conditions. Finally, conclusions are drawn in Section 6 .

\section{Preliminaries}

This section reviews some relevant basic concepts and lemmas. For $x \in \mathbb{R}, \operatorname{sgn}(x)$ denotes the standard sign function and $\operatorname{sig}^{\alpha}(x)$ is denoted by $\operatorname{sgn}(x)|x|^{\alpha}, \forall \alpha \geq 0$.

Consider the nonlinear autonomous system, depicted by

$$
\begin{aligned}
\dot{x} & =f(x), \\
x & \in D \subseteq \mathbb{R}^{n}, \\
f(0) & =0,
\end{aligned}
$$

where $f: \mathbb{R}^{n} \rightarrow \mathbb{R}^{n}$ is a continuous vector field on $x$. Under this condition, the definition of finite-time stability can be represented as follows.
Definition 1 (finite-time stability [18]). The equilibrium $x=0$ of system (1) is finite-time convergent if there is an open neighbourhood $U$ of the origin and a function $T_{x}: U \backslash$ $\{0\} \rightarrow(0, \infty)$, such that every solution trajectory $x\left(t, x_{0}\right)$ of system (1) starting from the initial point $x_{0} \in U \backslash\{0\}$ is welldefined and unique in forward time for $t \in\left[0, T_{x}\left(x_{0}\right)\right)$, and $\lim _{t \rightarrow T_{x}\left(x_{0}\right)} x\left(t, x_{0}\right)=0$. Here $T_{x}\left(x_{0}\right)$ is called the convergence time (with respect to the initial state $x_{0}$ ). The equilibrium of system (1) is finite-time stable if it is Lyapunov stable and finite-time convergent. If $U=D=\mathbb{R}^{n}$, the origin is a globally finite-time stable equilibrium.

Lemma 2 (see [19]). Let $k_{1}, \ldots, k_{n}>0$ be such that the polynomial $s^{n}+k_{n} s^{n-1}+\cdots+k_{1}$ is Hurwitz, and consider the system

$$
\begin{gathered}
\dot{x}_{1}=x_{2}, \\
\vdots \\
\dot{x}_{n-1}=x_{n}, \\
\dot{x}_{n}=u .
\end{gathered}
$$

There exists $\varepsilon \in(0,1)$ such that, for every $\alpha \in(1-\varepsilon, 1)$, the origin is a globally finite-time stable equilibrium for system (2) under the feedback control law:

$$
u=-k_{1} \operatorname{sig}^{\alpha_{1}}\left(x_{1}\right)-\cdots-k_{n} \operatorname{sig}^{\alpha_{n}}\left(x_{n}\right)
$$

where $\alpha_{1}, \ldots, \alpha_{n}$ satisfy

$$
\alpha_{i-1}=\frac{\alpha_{i} \alpha_{i+1}}{2 \alpha_{i+1}-\alpha_{i}}, \quad i=2, \ldots, n,
$$

with $\alpha_{n+1}=1$ and $\alpha_{n}=\alpha$.

\section{Modeling of Pneumatic Servo System}

In this section, we firstly introduce the model of pneumatic servo system and its operating principle. Figure 1 describes the schematic of a double-acting single-rod pneumatic servo system. The pneumatic servo system is combined by a proportional valve, a double-acting single-rod cylinder, two pressure sensors, a digital computer, a linear encoder, a decoder, an analog-to-digital converter, and a digital-to-analog converter. $P_{1}$ and $P_{2}$, respectively, represent the pressures inside the two chambers, and they are measured by pressure sensors. $A_{1}$ and $A_{2}$ are piston areas facing chamber 1 and chamber 2, respectively. In Figure 1, the pressure difference acting on the piston forces the piston/load to move, and the piston/load displacement is measured by the linear encoder. Next the displacement signal decoded by the decoder is fed back to the computer. Then, the control signal generated from computer is converted into servo valve, which determines the valve motion and thus determines the pressure inside the two chambers. Then, the dynamics of pneumatic cylinder is firstly modeled. 


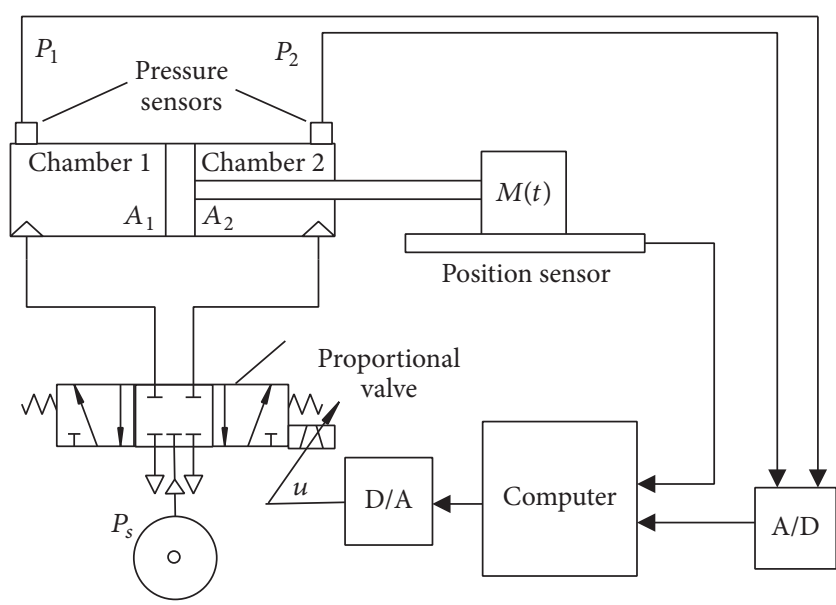

FIGURE 1: The schematic of pneumatic servo system.

3.1. Modeling of Pneumatic Cylinder. According to [32], the dynamics of cylinder pressures is modeled as follows:

$$
\begin{aligned}
\dot{P}_{1} & =-\frac{k P_{1} \dot{x}_{L}}{x_{L}}+\left(\sqrt{\frac{k}{R T_{s}}} \frac{R k T_{s} \gamma_{1 b} P_{s}(1+\operatorname{sgn}(u))}{2 A_{1} x_{L}}\right. \\
& \left.-\sqrt{\frac{k}{R T_{1}}} \frac{R k T_{1} \gamma_{1 e} P_{1}(1-\operatorname{sgn}(u))}{2 A_{1} x_{L}}\right)\left(\frac{A_{0 \max }}{\left|u_{\max }\right|}\right)|u| \\
& +d_{1}(t),
\end{aligned}
$$

$$
\begin{aligned}
\dot{P}_{2} & =\frac{k P_{2} \dot{x}_{L}}{L-x_{L}}+\left(\sqrt{\frac{k}{R T_{s}}} \frac{R k T_{s} \gamma_{2 b} P_{s}(1-\operatorname{sgn}(u))}{2 A_{2}\left(L-x_{L}\right)}\right. \\
& \left.-\sqrt{\frac{k}{R T_{2}}} \frac{R k T_{2} \gamma_{2 e} P_{2}(1+\operatorname{sgn}(u))}{2 A_{2}\left(L-x_{L}\right)}\right)\left(\frac{A_{0 \max }}{\left|u_{\max }\right|}\right)|u| \\
& +d_{2}(t)
\end{aligned}
$$

where $R, P_{s}$, and $P_{\text {atm }}$ are the gas constant, the source pressure, and the atmospheric pressure, respectively. $A_{0 \max }$ represents the largest area of orifice, $u_{\max }$ denotes the maximum control signal, $k=C_{p} / C_{v}$ is the specific heat ratio with the denotations of the constant-pressure specific heat $C_{p}$, and constant-volume specific heat of the air $C_{v} . T_{1}, T_{2}$, and $T_{s}$ are the temperature of chamber 1 , chamber 2 , and the source, respectively. $L$ is denoted by $l_{s}+x_{e 1}+x_{e 2}$, where $l_{s}$ is the stroke length, and $x_{e 1}$, and $x_{e 2}$ are the extra lengths of chamber 1 and chamber 2 , respectively. $d_{1}$ and $d_{2}$ represent external disturbances. We assume that there exist two positive constants $\bar{d}_{1}$ and $\bar{d}_{2}$ such that $\left|\dot{d}_{i}(t)\right| \leq \bar{d}_{i}, i=1,2$; that is, the derivatives of external disturbances $d_{1}(t)$ and $d_{2}(t)$ are all bounded. In practice, the above conditions are natural for the external disturbances. Let $x_{c}$ denote the real piston displacement; then, the effective piston displacement is $x_{L}=$ $x_{c}+x_{e 1}$ as shown by Figure 2. Parameter $\gamma_{1 b}$ is the modifying factor when chamber 1 is building the pressure, and $\gamma_{1 e}$ is the modifying factor when chamber 1 is exhausting the pressure. Parameter $\gamma_{2 b}$ is the modifying factor when chamber 2 is building the pressure, and $\gamma_{2 e}$ the modifying factor when chamber 2 is exhausting the pressure.

We define the four modifying factors as follows:

$$
\begin{aligned}
& \gamma_{i e}=\left\{\begin{array}{ll}
\sqrt{\frac{2}{k-1}}\left(\frac{P_{\mathrm{atm}}}{P_{i}}\right)^{(k+1) / 2 k} \sqrt{\left(\frac{P_{\mathrm{atm}}}{P_{i}}\right)^{(1-k) / k}-1,} & \frac{P_{\mathrm{atm}}}{P_{i}} \leq\left(\frac{2}{k+1}\right)^{k /(k-1)} \\
0.58, & \frac{P_{\mathrm{atm}}}{P_{i}}>\left(\frac{2}{k+1}\right)^{k /(k-1)},
\end{array} \quad i=1,2,\right. \\
& \gamma_{i b}=\left\{\begin{array}{ll}
\sqrt{\frac{2}{k-1}}\left(\frac{P_{i}}{P_{s}}\right)^{(k+1) / 2 k} \sqrt{\left(\frac{P_{i}}{P_{s}}\right)^{(1-k) / k}-1,} & \frac{P_{i}}{P_{s}} \leq\left(\frac{2}{k+1}\right)^{k /(k-1)} \\
0.58, & \frac{P_{i}}{P_{s}}>\left(\frac{2}{k+1}\right)^{k /(k-1)},
\end{array} \quad i=1,2 .\right.
\end{aligned}
$$

3.2. Dynamic Model of Pneumatic Servo System. The dynamics of the actuator piston are represented as the following form:

$$
\ddot{x}_{L}=\frac{P_{1} A_{1}}{M}-\frac{P_{2} A_{2}}{M},
$$

where $x_{L}$ and $M$ are the piston displacement and the load mass, respectively. With the denotations of $x_{1}=x_{L}, x_{2}=$ $\dot{x}_{1}, x_{3}=P_{1} A_{1}-P_{2} A_{2}$ and dynamic models (5)-(7) in mind, we obtain

$$
\begin{aligned}
& \dot{x}_{1}=x_{2}, \\
& \dot{x}_{2}=\frac{x_{3}}{M}, \\
& \dot{x}_{3}=F_{0}\left(x_{1}\right) x_{2}+F_{1}\left(x_{1}, u\right) u+d(t),
\end{aligned}
$$

where $F_{0}\left(x_{1}\right)=-k P_{1} A_{1} / x_{1}-k P_{2} A_{2} /\left(L-x_{1}\right), F_{1}\left(x_{1}, u\right)=$ $k \sqrt{R k}\left(A_{0 \max } /\left|u_{\max }\right|\right)\left\{[(\operatorname{sgn}(u)+1) / 2]\left(\sqrt{T_{s}} \gamma_{1 b} P_{s} / x_{1}+\right.\right.$ $\left.\sqrt{T_{2}} \gamma_{2 e} P_{2} /\left(L-x_{1}\right)\right)+[(1-\operatorname{sgn}(u)) / 2]\left(\sqrt{T_{1}} \gamma_{1 e} P_{1} / x_{1}+\right.$ $\left.\left.\sqrt{T_{s}} \gamma_{2 b} P_{s} /\left(L-x_{1}\right)\right)\right\}, d(t)=A_{1} d_{1}(t)-A_{2} d_{2}(t) \cdot x=\left[x_{1}\right.$, $\left.x_{2}, x_{3}\right]^{\mathrm{T}}$ denotes the state vector of system (8). $x_{d}$ represents 


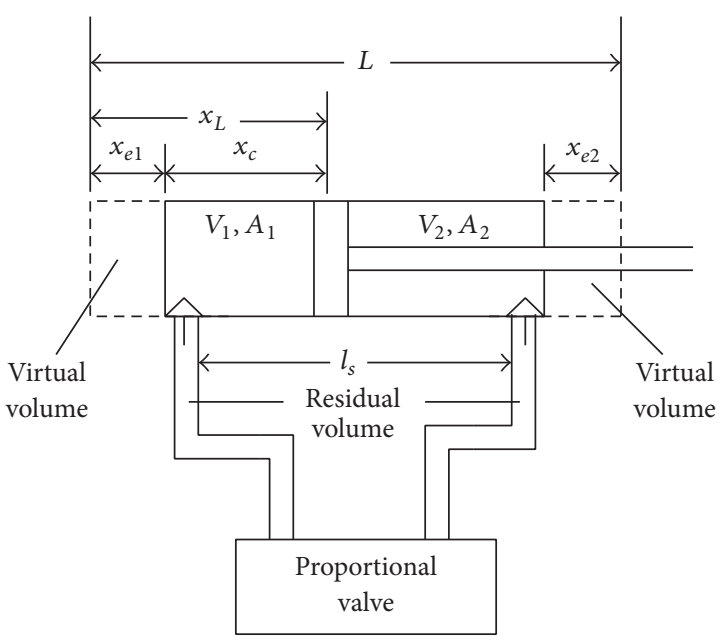

FIGURE 2: The parameters of the pneumatic cylinder.

the desired position of the piston, which satisfies that $x_{d}$ is third-order differentiable, and $x_{d}^{(3)}$ is bounded. In practice, lots of practical reference signals satisfy the above condition, such as step signals, ramp signals, polynomial signals, exponential signals and sinusoid signals, as well as their products and combinations.

Denoting the tracking errors $w_{1}=x_{d}-x_{1}, w_{2}=\dot{w}_{1}$, and $w_{3}=\dot{w}_{2}$, the tracking error system can be written as

$$
\begin{aligned}
\dot{w}_{1}= & w_{2}, \\
\dot{w}_{2}= & w_{3}, \\
\dot{w}_{3}= & x_{d}^{(3)}-\frac{F_{0}\left(x_{d}-w_{1}\right)\left(\dot{x}_{d}-w_{2}\right)}{M} \\
& \quad-\frac{F_{1}\left(x_{d}-w_{1}, u\right) u}{M}-\frac{d(t)}{M} .
\end{aligned}
$$

Then, the following control design will be conducted based on tracking error system (9).

\section{Composite Controller Design}

Controller design for tracking error system (9) is mainly composed of two parts, that is, finite-time disturbance observer design and composite controller design.

4.1. Finite-Time Disturbance Observer Design. According to [30], a finite-time disturbance observer for system (8) is designed as follows:

$$
\begin{aligned}
& \dot{\hat{x}}_{3}=F_{0}\left(x_{1}\right) x_{2}+F_{1}\left(x_{1}, u\right) u+v, \\
& v=-\lambda_{0} L^{1 / 2}\left|\widehat{x}_{3}-x_{3}\right|^{1 / 2} \operatorname{sgn}\left(\hat{x}_{3}-x_{3}\right)+\widehat{d}, \\
& \dot{\hat{d}}=-\lambda_{1} L \operatorname{sgn}(\widehat{d}-v),
\end{aligned}
$$

where $\lambda_{0}$ and $\lambda_{1}$ are the observer coefficients to be designed and $\hat{x}_{3}$ and $\widehat{d}$ are the estimates of $x_{3}$ and $d$, respectively. Then, combing (8) with (10), the observer estimation error is governed by

$$
\begin{aligned}
& \dot{e}_{3}=-\lambda_{0} L^{1 / 2}\left|e_{3}\right|^{1 / 2} \operatorname{sgn}\left(e_{3}\right)+e_{d}, \\
& \dot{e}_{d}=-\lambda_{1} L \operatorname{sgn}\left(\lambda_{0} L^{1 / 2}\left|e_{3}\right|^{1 / 2} \operatorname{sgn}\left(e_{3}\right)\right)-\dot{d},
\end{aligned}
$$

where the estimation errors are defined as $e_{3}=\widehat{x}_{3}-x_{3}$ and $e_{d}=\widehat{d}-d$.

Since there exist two positive constants $\bar{d}_{1}$ and $\bar{d}_{2}$ such that $\left|\dot{d}_{i}(t)\right| \leq \bar{d}_{i}, \quad i=1,2$, it can be obtained that $\dot{d}$ is bounded. It follows from [30] that the observer error system (11) is finite-time stable, which means that there exists a finite time $t_{1}$ such that the estimate errors converge to zero while $t \geq t_{1}$.

4.2. Composite Controller Design. First of all, a novel dynamic sliding mode manifold is defined by

$$
s=w_{3}-\int_{0}^{t} u^{*} d \tau,
$$

where $u^{*}$ is a nonsmooth controller which globally finitetime stabilizes the following system:

$$
\begin{aligned}
& \dot{w}_{1}=w_{2} \\
& \dot{w}_{2}=w_{3}, \\
& \dot{w}_{3}=u^{*}
\end{aligned}
$$

Then, by means of homogeneous theory, a detailed finitetime composite controller design process is presented by the following theorem.

Theorem 3 (consider the system (9)). If the controller $u$ is designed to satisfy the following condition:

$$
\begin{aligned}
F_{1}\left(x_{d}-w_{1}, u\right) u= & M x_{d}^{(3)}-F_{0}\left(x_{d}-w_{1}\right)\left(\dot{x}_{d}-w_{2}\right) \\
& -\widehat{d}-M u^{*}+M \eta \operatorname{sgn}(s),
\end{aligned}
$$

where $u^{*}=-k_{1} \operatorname{sig}^{\alpha_{1}}\left(w_{1}\right)-k_{2} \operatorname{sig}^{\alpha_{2}}\left(w_{2}\right)-k_{3} \operatorname{sig}^{\alpha_{3}}\left(w_{3}\right), k_{i}$, $\alpha_{i}, i=1,2,3$ are constants satisfying the conditions given in Lemma $2, \eta>0$, and the sliding mode manifold $s$ is defined as (12), then the closed-loop systems (9) and (14) are globally finite-time stable; namely, the tracking errors will be stabilized to zero in finite time. Moreover, the proposed controller can be decoupled from the practical point of view. Controller (14) can be described in a decoupling form as follows: 


$$
u= \begin{cases}\frac{Z}{k \sqrt{R k}\left(A_{0 \max } /\left|u_{\max }\right|\right)\left(\sqrt{T_{s}} \gamma_{1 b} P_{s} / x_{1}+\sqrt{T_{2}} \gamma_{2 e} P_{2} /\left(L-x_{1}\right)\right)}, & Z \geq 0, \\ \frac{Z}{k \sqrt{R k}\left(A_{0 \max } /\left|u_{\max }\right|\right)+\left(\sqrt{T_{1}} \gamma_{1 e} P_{1} / x_{1}+\sqrt{T_{s}} \gamma_{2 b} P_{s} /\left(L-x_{1}\right)\right)}, & Z<0,\end{cases}
$$

where $Z=M x_{d}^{(3)}-F_{0}\left(x_{d}-w_{1}\right)\left(\dot{x}_{d}-w_{2}\right)-\widehat{d}-M u^{*}+M \eta \operatorname{sgn}(s)$.

Proof. The stability analysis can be divided into two steps. In Step 1, the states of system (13) will not escape to infinity for $t \leq t_{1}$. In Step 2, system (13) is finite-stable when $t \leq t_{1}$.

Step 1. An energy function is defined as

$$
\begin{aligned}
& B\left(x_{1}, x_{2}, x_{3}, w_{1}, w_{2}, w_{3}\right) \\
& \quad=x_{1}^{2}+x_{2}^{2}+x_{3}^{2}+w_{1}^{2}+w_{2}^{2}+w_{3}^{2} .
\end{aligned}
$$

Since the desired position signal $x_{d}$ is third-order differentiable and $d$ is estimated by $\widehat{d}$ in finite time $t_{1}$, taking the derivative of $B$ yields

$$
\begin{aligned}
\dot{B} & =2 x_{1} \dot{x}_{1}+2 x_{2} \dot{x}_{2}+2 x_{3} \dot{x}_{3}+2 w_{1} \dot{w}_{1}+2 w_{2} \dot{w}_{2} \\
& +2 w_{3} \dot{w}_{3}=2 x_{1} x_{2}+\frac{2 x_{2} x_{3}}{M}+2 x_{3}\left(F_{0}\left(x_{1}\right) x_{2}\right. \\
& \left.+F_{1}\left(x_{1}, u\right) u+d(t)\right)+2 w_{1} w_{2}+2 w_{2} w_{3} \\
& +2 w_{3}\left(x_{d}^{(3)}-\frac{F_{0}\left(x_{d}-w_{1}\right)\left(\dot{x}_{d}-w_{2}\right)}{M}\right. \\
& \left.-\frac{F_{1}\left(x_{d}-w_{1}, u\right) u}{M}-\frac{d(t)}{M}\right) \leq x_{1}^{2}+x_{2}^{2}+\frac{x_{2}^{2}+x_{3}^{2}}{M} \\
& +\left(M x_{d}^{(3)}+M \eta \operatorname{sgn}(s)-e_{d}\right)\left(1+x_{3}^{2}\right)+2 M x_{3} u^{*} \\
& +w_{1}^{2}+w_{2}^{2}+w_{2}^{2}+w_{3}^{2}+\left(-\eta \operatorname{sgn}(s)-\frac{e_{d}}{M}\right)(1 \\
& \left.+w_{3}^{2}\right)+2 w_{3} u^{*} .
\end{aligned}
$$

Since $x_{d}^{(3)}$ is bounded and the finite-time disturbance observer (10) is stable in a finite time, it can be obtained that there exists a positive constant $\bar{M}$ such that

$$
\begin{aligned}
& \bar{M}=\max \left\{\sup \left(M x_{d}^{(3)}+M \eta \operatorname{sgn}(s)-e_{d}\right),\right. \\
& \left.\sup \left(-\eta \operatorname{sgn}(s)-\frac{e_{d}}{M}\right)\right\} .
\end{aligned}
$$

According to Lemma 2, we know

$$
u^{*}=-k_{1} \operatorname{sig}^{\alpha_{1}}\left(w_{1}\right)-k_{2} \operatorname{sig}^{\alpha_{2}}\left(w_{2}\right)-k_{3} \operatorname{sig}^{\alpha_{3}}\left(w_{3}\right)
$$

where $\alpha_{1}, \alpha_{2}$, and $\alpha_{3}$ satisfy

$$
\alpha_{i-1}=\frac{\alpha_{i} \alpha_{i+1}}{2 \alpha_{i+1}-\alpha_{i}}, \quad i=2,3
$$

with $\alpha_{4}=1$ and $\alpha_{3}=\alpha$. Then, it can be obtained that

$$
\begin{aligned}
& \alpha_{1}=\frac{\alpha}{3-2 \alpha}, \\
& \alpha_{2}=\frac{\alpha}{2-\alpha}
\end{aligned}
$$

which means that $0<\alpha_{i}<1(i=1,2,3)$ hold. Hence, the following inequalities can be established:

$$
\begin{aligned}
& \left|x_{3} u^{*}\right|=\mid x_{3}\left(-k_{1} \operatorname{sig}^{\alpha_{1}}\left(w_{1}\right)-k_{2} \operatorname{sig}^{\alpha_{2}}\left(w_{2}\right)\right. \\
& \left.-k_{3} \operatorname{sig}^{\alpha_{3}}\left(w_{3}\right)\right)|\leq| x_{3} \mid\left(\left|k_{1}\right|\left(\left|x_{1}\right|+1\right)+\left|k_{2}\right|\left(\left|x_{2}\right|\right.\right. \\
& \left.+1)+\left|k_{3}\right|\left(\left|x_{3}\right|+1\right)\right) \leq \frac{\left|k_{1}\right|}{2}\left(2 x_{3}^{2}+w_{1}^{2}+1\right) \\
& +\frac{\left|k_{2}\right|}{2}\left(2 x_{3}^{2}+w_{2}^{2}+1\right)+\frac{\left|k_{3}\right|}{2}\left(2 x_{3}^{2}+w_{3}^{2}+1\right) \\
& =\left(\left|k_{1}\right|+\left|k_{2}\right|+\left|k_{3}\right|\right) x_{3}^{2}+\frac{\left|k_{1}\right|}{2} w_{1}^{2}+\frac{\left|k_{2}\right|}{2} w_{2}^{2}+\frac{\left|k_{3}\right|}{2} \\
& \cdot w_{3}^{2}+\frac{\left|k_{1}\right|}{2}+\frac{\left|k_{2}\right|}{2}+\frac{\left|k_{3}\right|}{2} \\
& \left|w_{3} u^{*}\right|=\mid w_{3}\left(-k_{1} \operatorname{sig}^{\alpha_{1}}\left(w_{1}\right)-k_{2} \operatorname{sig}^{\alpha_{2}}\left(w_{2}\right)\right. \\
& \left.-k_{3} \operatorname{sig}^{\alpha_{3}}\left(w_{3}\right)\right)|\leq| w_{3} \mid\left(\left|k_{1}\right|\left(\left|x_{1}\right|+1\right)+\left|k_{2}\right|\left(\left|x_{2}\right|\right.\right. \\
& \left.+1)+\left|k_{3}\right|\left(\left|x_{3}\right|+1\right)\right) \leq \frac{\left|k_{1}\right|}{2}\left(2 w_{3}^{2}+w_{1}^{2}+1\right) \\
& +\frac{\left|k_{2}\right|}{2}\left(2 w_{3}^{2}+w_{2}^{2}+1\right)+\frac{\left|k_{3}\right|}{2}\left(2 w_{3}^{2}+w_{3}^{2}+1\right) \\
& =\left(\left|k_{1}\right|+\left|k_{2}\right|+\frac{3\left|k_{3}\right|}{2}\right) w_{3}^{2}+\frac{\left|k_{1}\right|}{2} w_{1}^{2}+\frac{\left|k_{2}\right|}{2} w_{2}^{2} \\
& +\frac{\left|k_{1}\right|}{2}+\frac{\left|k_{2}\right|}{2}+\frac{\left|k_{3}\right|}{2} \text {. }
\end{aligned}
$$

Then, this yields

$$
\dot{B} \leq K B+K,
$$

where $K=\max \left\{\left|k_{1}\right|+\left|k_{2}\right|+3\left|k_{3}\right| / 2, M\left(\left|k_{1}\right|+\left|k_{2}\right|+\left|k_{3}\right|\right), 1 / 2+\right.$ $1 / 2 M,(1 / 2)(\bar{M}+1 / M), 1,(1 / 2) \bar{M}\}$. Solving inequality (24), 
it can be obtained that $B \leq(B(0)+1) e^{K t}-1$. Hence, system states are bounded when $t \leq t_{1}$.

Step 2. Taking the derivative of the sliding mode surface (12) yields

$$
\begin{aligned}
\dot{s}= & \dot{w}_{3}-u^{*} \\
= & x_{d}^{(3)}-\frac{F_{0}\left(x_{d}-w_{1}\right)\left(\dot{x}_{d}-w_{2}\right)}{M} \\
& -\frac{F_{1}\left(x_{d}-w_{1}, u\right) u}{M}-\frac{d(t)}{M}-u^{*} .
\end{aligned}
$$

According to (14), we obtain

$$
s \dot{s} \leq-\eta \operatorname{sgn}(s) s=-\eta|s|
$$

which implies that the states will converge to the sliding mode manifold $s=0$ in finite time. In addition, when the states reach the sliding mode manifold, it can be easily concluded that

$$
\dot{s}=\dot{w}_{3}-u^{*}=0
$$

It is clear that (27) implies

$$
\begin{aligned}
& \dot{w}_{1}=w_{2}, \\
& \dot{w}_{2}=w_{3}, \\
& \dot{w}_{3}=u^{*}
\end{aligned}
$$

Thus, we know that the states of system (13) finitely converge to zero.

Remark 4. It is highlighted that the proposed composite controller (14) consists of two parts including a baseline nonsmooth state feedback and a disturbance compensation; such a control strategy has nice robustness against external disturbances due to its nonsmooth character and disturbance compensation, and the proposed finite-time composite controller provides better position tracking performance and better disturbance rejection property than the pure integral sliding mode controller (ISMC), which is designed as

$$
\begin{gathered}
F_{1}\left(x_{d}-w_{1}, u\right) u=M x_{d}^{(3)}-F_{0}\left(x_{d}-w_{1}\right)\left(\dot{x}_{d}-w_{2}\right) \\
-M u^{*}+M \eta \operatorname{sgn}(s),
\end{gathered}
$$

where $u^{*}=-k_{1} \operatorname{sig}^{\alpha_{1}}\left(w_{1}\right)-k_{2} \operatorname{sig}^{\alpha_{2}}\left(w_{2}\right)-k_{3} \operatorname{sig}^{\alpha_{3}}\left(w_{3}\right)$; the controller (29) is represented in a decoupling form as follows. The controller (14) can be described in a decoupling form as follows:

$$
u=\left\{\begin{array}{cl}
\frac{Z}{k \sqrt{R k}\left(A_{0 \max } /\left|u_{\max }\right|\right)\left(\sqrt{T_{s}} \gamma_{1 b} P_{s} / x_{1}+\sqrt{T_{2}} \gamma_{2 e} P_{2} /\left(L-x_{1}\right)\right)}, & Z \geq 0, \\
\frac{Z}{k \sqrt{R k}\left(A_{0 \max } /\left|u_{\max }\right|\right)+\left(\sqrt{T_{1}} \gamma_{1 e} P_{1} / x_{1}+\sqrt{T_{s}} \gamma_{2 b} P_{s} /\left(L-x_{1}\right)\right)}, & Z<0,
\end{array}\right.
$$

where $Z=M x_{d}^{(3)}-F_{0}\left(x_{d}-w_{1}\right)\left(\dot{x}_{d}-w_{2}\right)-M u^{*}+M \eta \operatorname{sgn}(s)$. It is obvious that the ISMC (29) is similar to (14), but there is no disturbance compensation $\widehat{d}$ in ISMC, which leads to the better disturbance rejection property of the proposed finitetime composite controller.

\section{Numerical Simulations}

In order to validate the effectiveness of the proposed composite control scheme, this section presents some results of numerical simulations compared with ISMC (29). Table 1 shows the model parameters of the pneumatic servo system.

The desired position is set as $0.5 \mathrm{~m}$, and a desired transient profile is designed as follows:

$$
x_{d}(t)=0.5+0.05 \sin (\pi t) .
$$

To have a fair comparison, the control signals are limited not to exceed $u_{\max }=5 \mathrm{~V}$. Considerable effects have been devoted to regulating the performances of both closed-loop systems as good as possible.
Case 1 (constant disturbances). The disturbances are chosen as $d_{1}=d_{2}=2 \times 10^{5}$ in this case. The parameters of controller (14) are chosen as $k_{1}=220, k_{2}=450, k_{3}=350, \alpha_{1}=$ $4 / 7, \alpha_{2}=2 / 3, \alpha_{3}=4 / 5, \eta=20, L=5, \lambda_{0}=10$, and $\lambda_{1}=$ 5 and the parameters of ISMC are chosen as $l_{1}=200, l_{2}=$ 500 , and $l_{3}=400, \beta_{1}=3 / 4, \beta_{2}=9 / 11, \beta_{3}=9 / 10, \eta=$ 20. The sampling time is $0.01 \mathrm{~s}$. The simulation results are shown in Figures 3-5.

From Figure 3, it can be seen that the response cures of piston position, velocity, and their tracking errors converge to the origin in a finite time for both cases. However, the proposed composite controller has faster convergence rate since it combines with finite-time disturbance observer. It can be seen from Figure 4 that the disturbance $d$ and state $x_{3}$ estimate errors can converge to zero in a finite time. Figure 5 shows the control signal cures of the pneumatic servo system under the different controllers. According to the above analysis, it can be concluded that the proposed composite controller has a faster convergence rate and a better disturbance rejection property. 


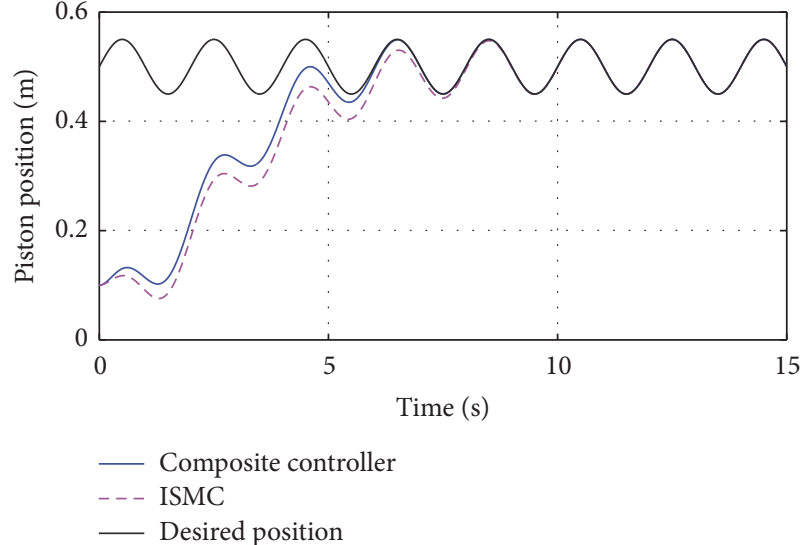

(a)

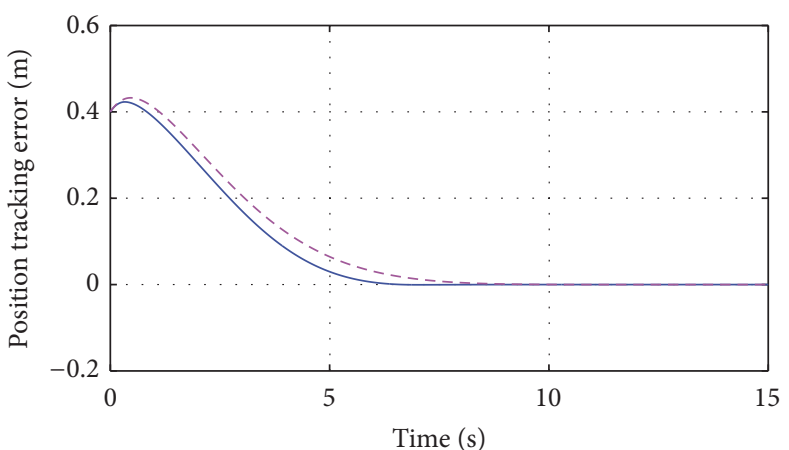

- Composite controller - - - ISMC

(c)

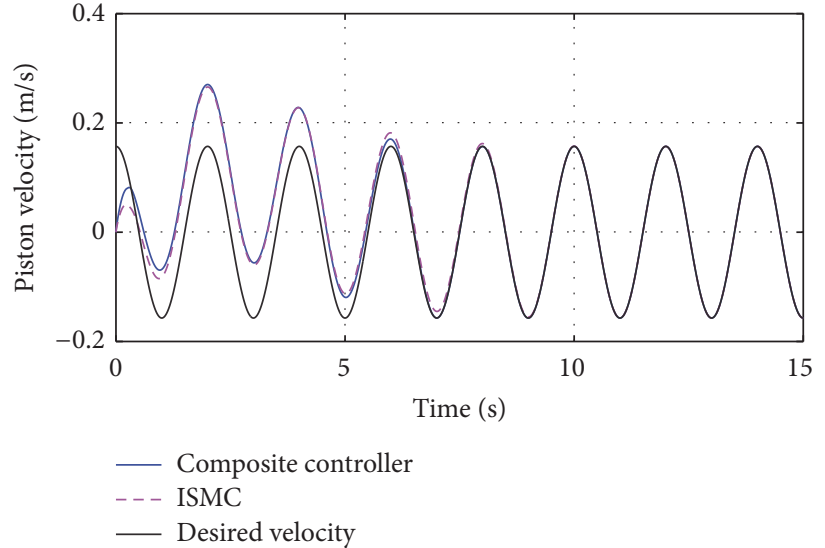

(b)

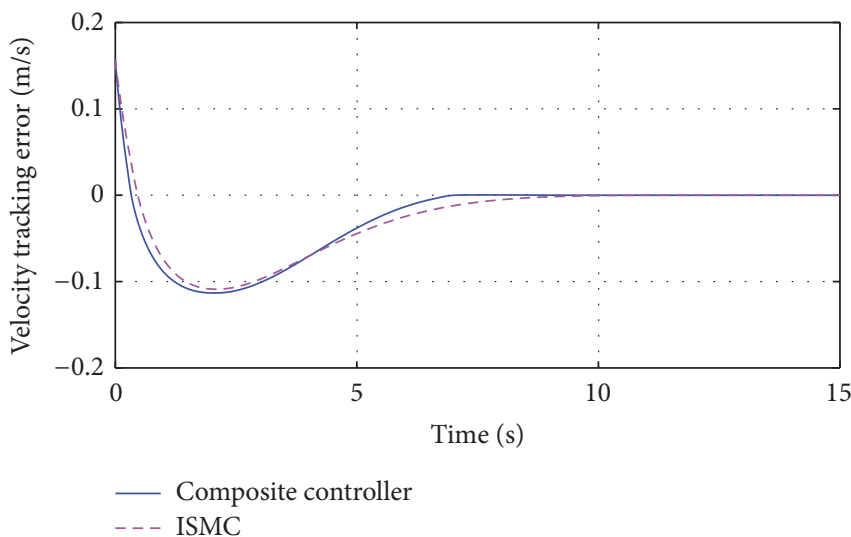

(d)

FIGURE 3: The response curves and tracking errors of the pneumatic servo system under the proposed composite controller and ISMC. (a) Piston position; (b) piston velocity; (d) piston position tracking error; (d) piston velocity tracking error.

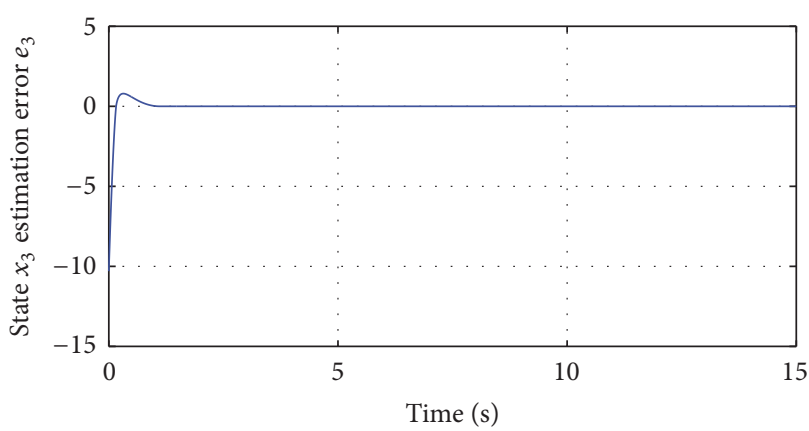

(a)

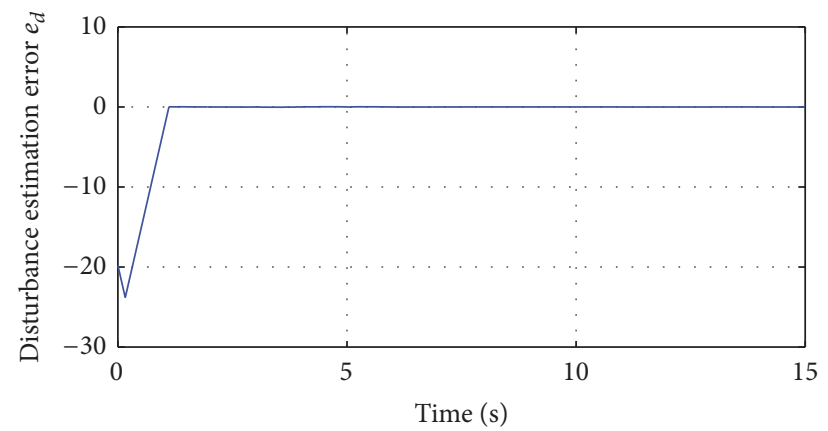

(b)

Figure 4: The estimation error curves of the pneumatic servo system under the proposed composite controller. (a) State $x_{3}$ estimation error $e_{3}$; (b) disturbance $d$ estimation error $e_{d}$.

Case 2 (time-varying disturbances). In this case, the disturbances are selected as $d_{1}=10^{5}(1+\sin (t))$ and $d_{2}=10^{5}(1+$ $\cos (t))$. The parameters of controller (14) are chosen as $k_{1}=$ $220, k_{2}=450, k_{3}=360, \alpha_{1}=4 / 7, \alpha_{2}=2 / 3, \alpha_{3}=$ $4 / 5, \eta=20, L=500, \lambda_{0}=10$, and $\lambda_{1}=5$ and the parameters of ISMC are chosen as $l_{1}=200, l_{2}=500, l_{3}=$ 400, $\beta_{1}=3 / 4, \beta_{2}=9 / 11, \beta_{3}=9 / 10$, and $\eta=20$. The sampling time is $0.01 \mathrm{~s}$. The simulation results are shown in Figures 6-8.

Figure 6 shows the response cures of piston position, velocity, and their tracking errors; it can be observed that the response cures converge to the origin in a finite time for both cases. However, the proposed composite controller 


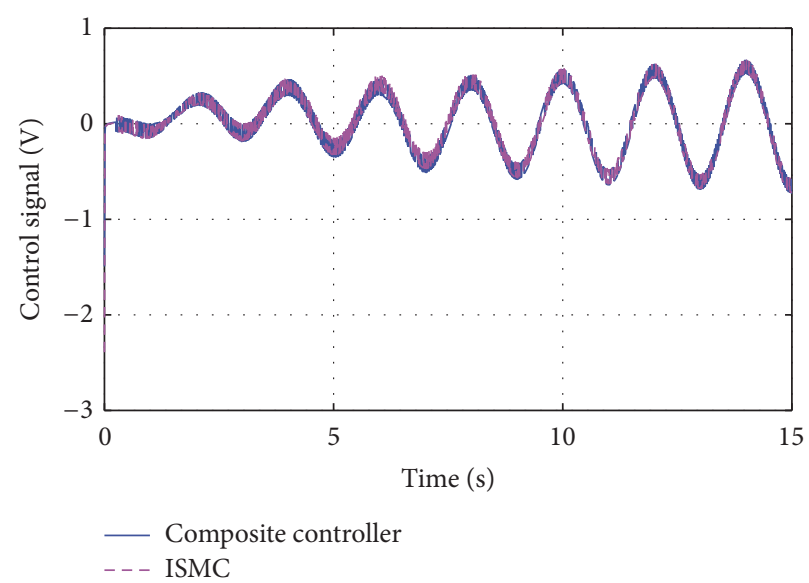

FIGURE 5: The control input curves of the pneumatic servo system under the proposed composite controller and ISMC.

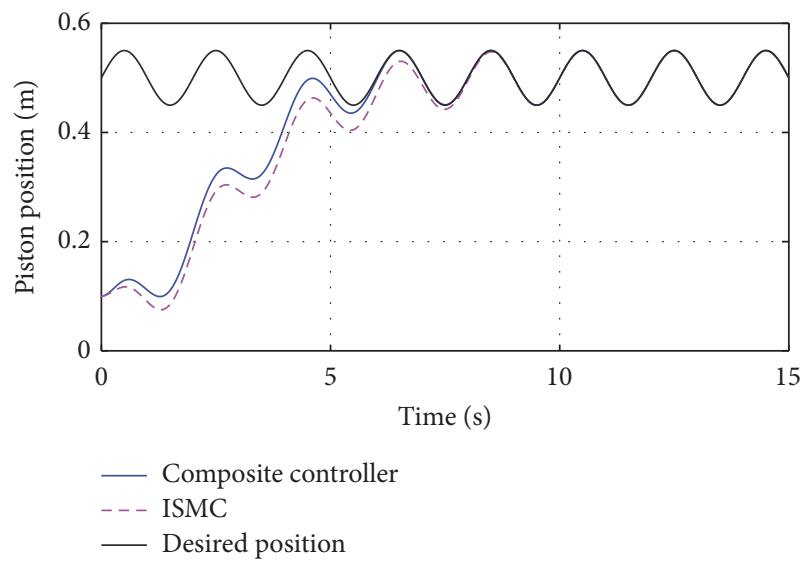

(a)

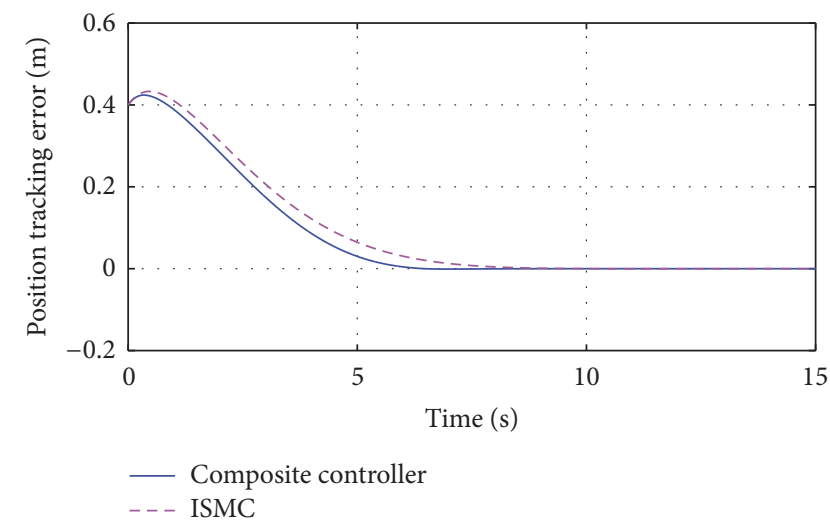

(c)

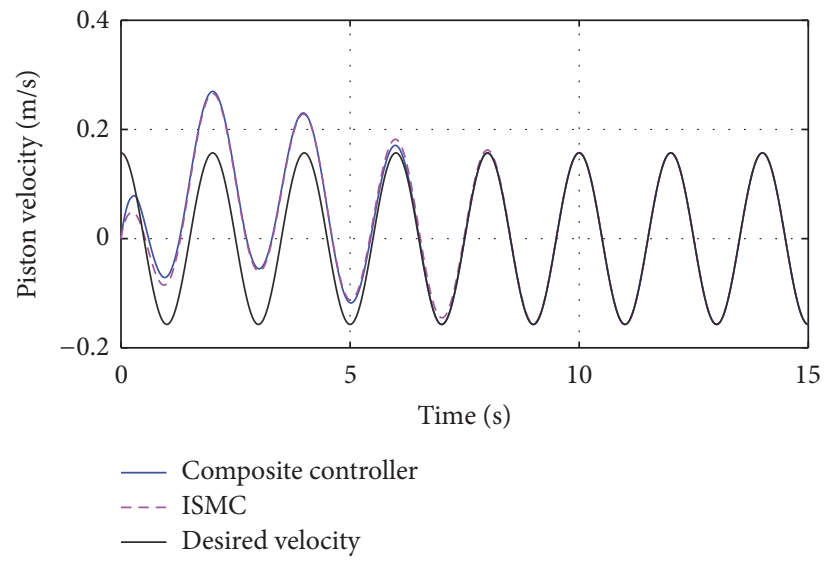

(b)

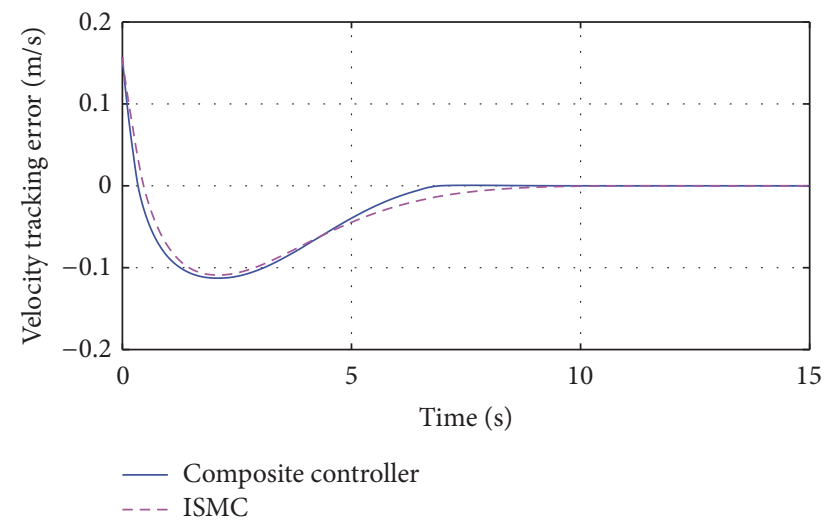

(d)

FIGURE 6: The response curves and tracking errors of the pneumatic servo system under the proposed composite controller and ISMC. (a) Piston position; (b) piston velocity; (c) piston position tracking error; (d) piston velocity tracking error.

has faster converge rate due to disturbance compensation. The disturbance estimate error and state estimate error can converge to finitely zero in Figure 7 . Figure 8 presents the control signal cures of the pneumatic servo system under the composite controller and the ISMC. As a consequence, the effectiveness of the proposed composite controller is verified.

\section{Conclusions}

This paper has studied the position tracking control problem of a pneumatic servo system. By utilizing sliding mode control and homogeneous theory, a composite controller with a finite-time disturbance observer has been proposed, 


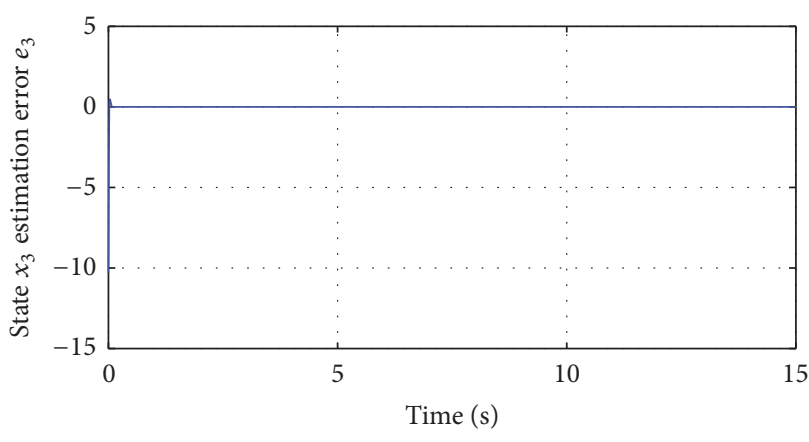

(a)

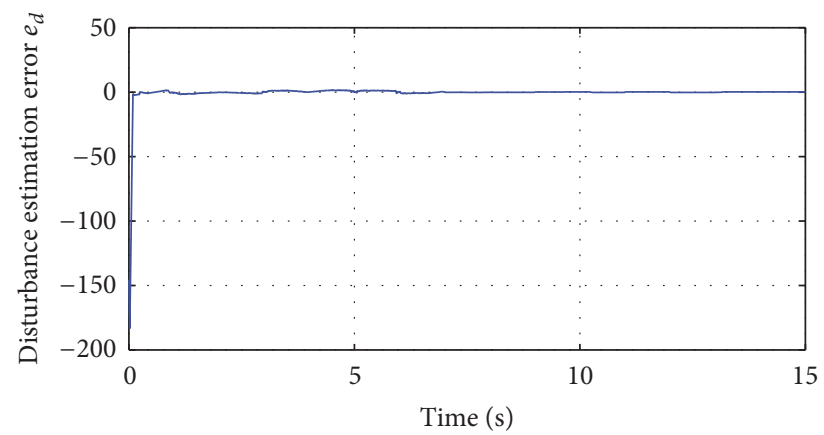

(b)

FIGURE 7: The estimation error curves of the pneumatic servo system under the proposed composite controller. (a) State $x_{3}$ estimation error $e_{3}$; (b) disturbance $d$ estimation error $e_{d}$.

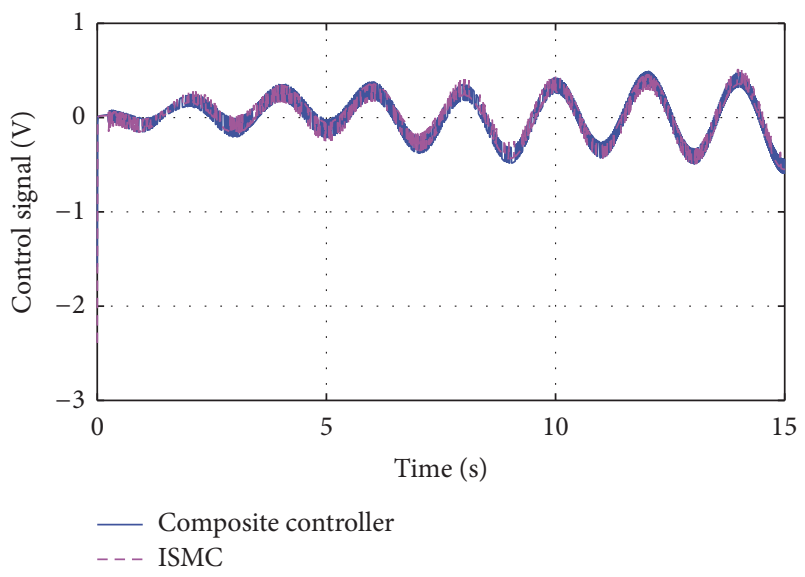

FIGURE 8: The control input curves of the pneumatic servo system under the proposed composite controller and ISMC.

TABLE 1: Model parameters of the pneumatic servo system.

\begin{tabular}{lc}
\hline Parameters & Values \\
\hline$k$ & 1.4 \\
$u_{\max }$ & $5 \mathrm{~V}$ \\
$M$ & $15 \mathrm{~kg}$ \\
$T_{1}$ & $293 \mathrm{~K}$ \\
$T_{2}$ & $293 \mathrm{~K}$ \\
$T_{s}$ & $293 \mathrm{~K}$ \\
$L$ & $1.029 \mathrm{~m}^{2}$ \\
$R$ & $286.987 \mathrm{~J} / \mathrm{kgK}$ \\
$x_{e 1}$ & $0.0132 \mathrm{~m}$ \\
$x_{e 2}$ & $0.0158 \mathrm{~m}$ \\
$P_{s}$ & $600000 \mathrm{~Pa}$ \\
$P_{\mathrm{atm}}$ & $101000 \mathrm{~Pa}$ \\
$A_{1}$ & $0.0012566 \mathrm{~m}^{2}$ \\
$A_{2}$ & $0.0010566 \mathrm{~m}^{2}$ \\
$A_{0 \max }$ & $0.000028274 \mathrm{~m}^{2}$ \\
\hline
\end{tabular}

which globally finite-time stabilizes the tracking error system. The proposed control scheme has nice robustness against external disturbances due to its nonsmooth character and disturbance compensation, and can effectively deal with hard nonlinearities by decoupling. The numerical simulations have shown that for the pneumatic servo system, the proposed nonsmooth composite controller provides better position tracking performances, that is, a faster tracking rate, a higher tracking accuracy, and a better disturbance rejection property.

\section{Competing Interests}

The authors declare that they have no competing interests.

\section{Acknowledgments}

This work was supported in part by the Priority Academic Program Development of Jiangsu Higher Education Institutions, Cooperative Innovation Funds of Jiangsu Province (BY2014127-09).

\section{References}

[1] Y. M. T. Elobaid, J. Huang, and Y. Wang, "Nonlinear disturbance observer based robust tracking control of pneumatic muscle," Mathematical Problems in Engineering, vol. 2014, Article ID 872093, 8 pages, 2014.

[2] T. Choi, B. Choi, and K. Seo, "Nonlinear disturbance observerbased dynamic surface control for trajectory tracking of pneumatic muscle system," IEEE Transactions on Control Systems Technology, vol. 22, no. 2, pp. 440-445, 2014.

[3] M. Chou and X. Xia, "Optimal cruise control of heavy-haul trains equipped with electronically controlled pneumatic brake systems," Control Engineering Practice, vol. 15, no. 5, pp. 511-519, 2007.

[4] T.-Y. Choi, B.-S. Choi, and K.-H. Seo, "Position and compliance control of a pneumatic muscle actuated manipulator for enhanced safety," IEEE Transactions on Control Systems Technology, vol. 19, no. 4, pp. 832-842, 2011.

[5] G. Li, X. Wang, and S. Li, "Finite-time state observer design for a pneumatic servo system," in Proceedings of the 27th Chinese Control and Decision Conference (CCDC'15), pp. 837-842, IEEE, Qingdao, China, May 2015. 
[6] D. Yuan and Z. Zhang, "Modelling and control scheme of the ball-plate trajectory-tracking pneumatic system with a touch screen and a rotary cylinder," IET Control Theory and Applications, vol. 4, no. 4, pp. 573-589, 2010.

[7] S. Riachy and M. Ghanes, "A nonlinear controller for pneumatic servo systems: design and experimental tests," IEEE/ASME Transactions on Mechatronics, vol. 19, no. 4, pp. 1363-1373, 2014.

[8] G. M. Bone and S. Ning, "Experimental comparison of position tracking control algorithms for pneumatic cylinder actuators," IEEE/ASME Transactions on Mechatronics, vol. 12, no. 5, pp. 557-561, 2007.

[9] T. Nguyen, J. Leavitt, F. Jabbari, and J. E. Bobrow, "Accurate Sliding-mode control of pneumatic systems using low-cost solenoid valves," IEEE/ASME Transactions on Mechatronics, vol. 12, no. 2, pp. 216-219, 2007.

[10] M. Taleb, A. Levant, and F. Plestan, "Pneumatic actuator control: Solution based on adaptive twisting and experimentation," Control Engineering Practice, vol. 21, no. 5, pp. 727-736, 2013.

[11] L. Zhao, Y. Yang, Y. Xia, and Z. Liu, "Active disturbance rejection position control for a magnetic rodless pneumatic cylinder," IEEE Transactions on Industrial Electronics, vol. 62, no. 9, pp. 5838-5846, 2015.

[12] A. Estrada and F. Plestan, "Second order sliding mode output feedback control with switching gains-application to the control of a pneumatic actuator," Journal of the Franklin Institute, vol. 351, no. 4, pp. 2335-2355, 2014.

[13] J. H. Lilly, "Adaptive tracking for pneumatic muscle actuators in bicep and tricep configurations," IEEE Transactions on Neural Systems and Rehabilitation Engineering, vol. 11, no. 3, pp. 333339, 2003.

[14] L.-W. Lee and I.-H. Li, "Wavelet-based adaptive sliding-mode control with $H_{\infty}$ tracking performance for pneumatic servo system position tracking control," IET Control Theory and Applications, vol. 6, no. 11, pp. 1699-1714, 2012.

[15] J. Sun, J. Yang, W. X. Zheng, and S. Li, "GPIO-based robust control of nonlinear uncertain systems under time-varying disturbance with application to DC-DC converter," IEEE Transactions on Circuits and Systems II: Express Briefs, vol. 63, no. 11, pp. 1074-1078, 2016.

[16] J. Sun and S. Li, "Disturbance observer based iterative learning control method for a class of systems subject to mismatched disturbances," Transactions of the Institute of Measurement and Control, 2016.

[17] S. Li, H. Du, and X. Lin, "Finite-time consensus algorithm for multi-agent systems with double-integrator dynamics," Automatica, vol. 47, no. 8, pp. 1706-1712, 2011.

[18] S. P. Bhat and D. S. Bernstein, "Finite-time stability of continuous autonomous systems," SIAM Journal on Control and Optimization, vol. 38, no. 3, pp. 751-766, 2000.

[19] S. P. Bhat and D. S. Bernstein, "Geometric homogeneity with applications to finite-time stability," Mathematics of Control, Signals, and Systems, vol. 17, no. 2, pp. 101-127, 2005.

[20] Q. Lan, S. Li, S. Khoo, and P. Shi, "Global finite-time stabilisation for a class of stochastic nonlinear systems by output feedback," International Journal of Control, vol. 88, no. 3, pp. 494-506, 2015.

[21] Y. Hong, Y. Xu, and J. Huang, "Finite-time control for robot manipulators," Systems \& Control Letters, vol. 46, no. 4, pp. $243-$ 253, 2002.

[22] S. Li, X. Wang, and L. Zhang, "Finite-time output feedback tracking control for autonomous underwater vehicles," IEEE Journal of Oceanic Engineering, vol. 40, no. 3, pp. 727-751, 2015.
[23] S. Ding, S. Li, and W. X. Zheng, "Nonsmooth stabilization of a class of nonlinear cascaded systems," Automatica, vol. 48, no. 10, pp. 2597-2606, 2012.

[24] X. Wang, X. Sun, S. Li, and H. Ye, "Finite-time position tracking control of rigid hydraulic manipulators based on highorder terminal sliding mode," Proceedings of the Institution of Mechanical Engineers. Part I: Journal of Systems and Control Engineering, vol. 226, no. 3, pp. 394-414, 2012.

[25] X. Wang, X. Sun, S. Li, and H. Ye, "Output feedback domination approach for finite-time force control of an electrohydraulic actuator," IET Control Theory \& Applications, vol. 6, no. 7, pp. 921-934, 2012.

[26] W.-H. Chen, J. Yang, L. Guo, and S. Li, "Disturbance observerbased control and related methods: an overview," IEEE Transactions on Industrial Electronics, vol. 63, no. 2, pp. 1083-1095, 2016.

[27] S. Li, J. Yang, W. Chen, and X. Chen, Disturbance ObserverBased Control: Methods and Applications, CRC Press, Boca Raton, Fla, USA, 2014.

[28] W.-H. Chen, D. J. Ballance, P. J. Gawthrop, and J. O’Reilly, “A nonlinear disturbance observer for robotic manipulators," IEEE Transactions on Industrial Electronics, vol. 47, no. 4, pp. 932-938, 2000.

[29] L. Guo and W.-H. Chen, "Disturbance attenuation and rejection for systems with nonlinearity via DOBC approach," International Journal of Robust and Nonlinear Control, vol. 15, no. 3, pp. 109-125, 2005.

[30] Y. B. Shtessel, I. A. Shkolnikov, and A. Levant, "Smooth secondorder sliding modes: missile guidance application," Automatica, vol. 43, no. 8, pp. 1470-1476, 2007.

[31] Y. Hong, J. Huang, and Y. Xu, "On an output feedback finitetime stabilization problem," IEEE Transactions on Automatic Control, vol. 46, no. 2, pp. 305-309, 2001.

[32] Y.-C. Tsai and A.-C. Huang, "Multiple-surface sliding controller design for pneumatic servo systems," Mechatronics, vol. 18, no. 9, pp. 506-512, 2008. 


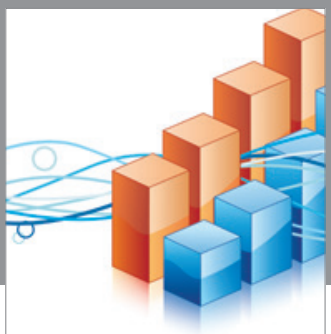

Advances in

Operations Research

vatem alat4

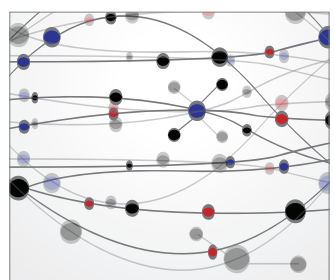

\section{The Scientific} World Journal
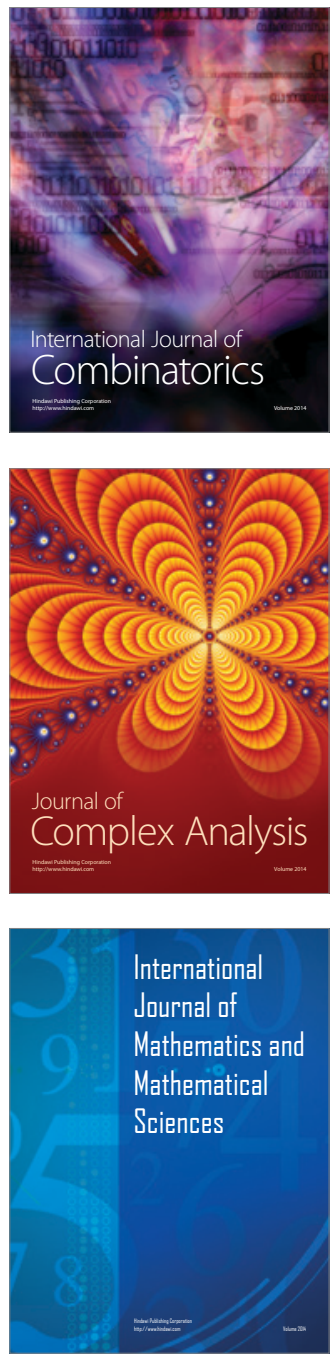
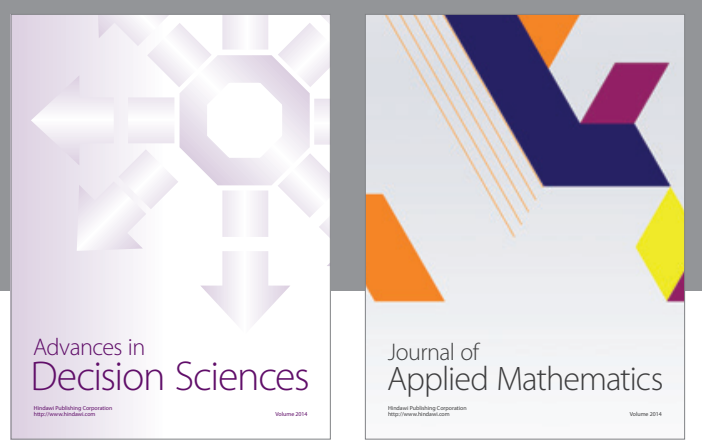

Algebra

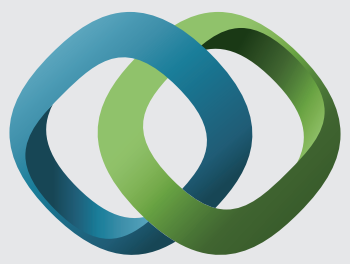

\section{Hindawi}

Submit your manuscripts at

http://www.hindawi.com
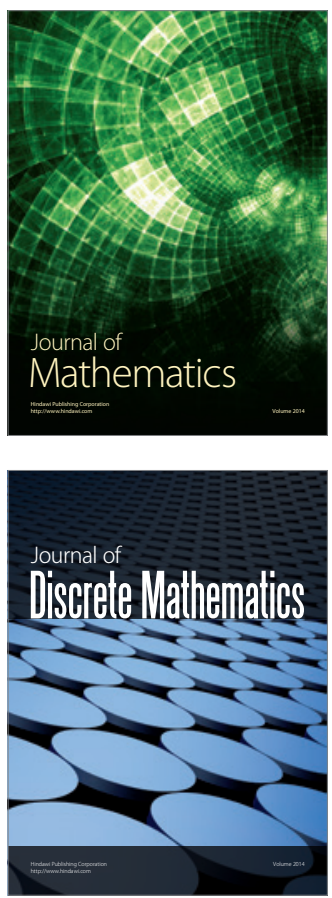

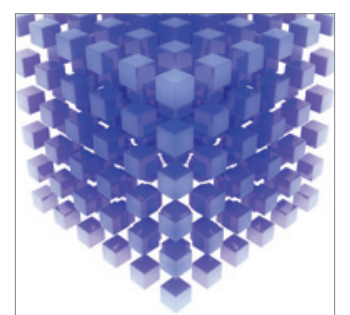

Mathematical Problems in Engineering
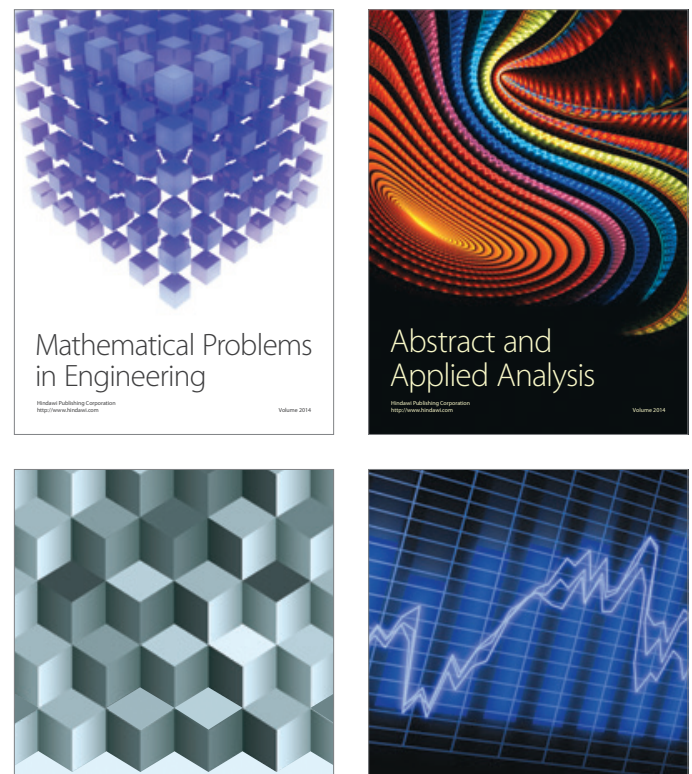

Journal of

Function Spaces

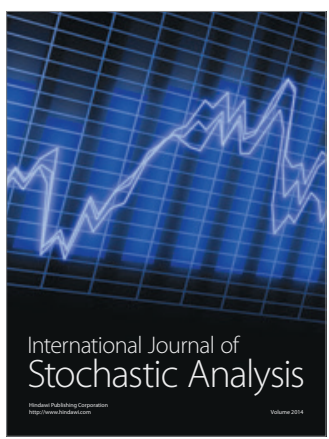

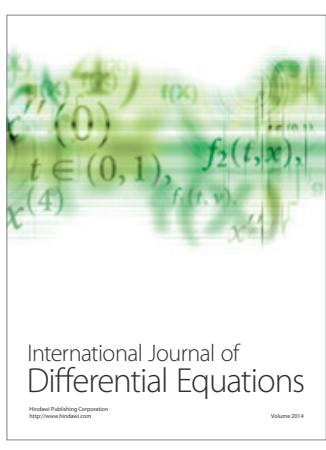
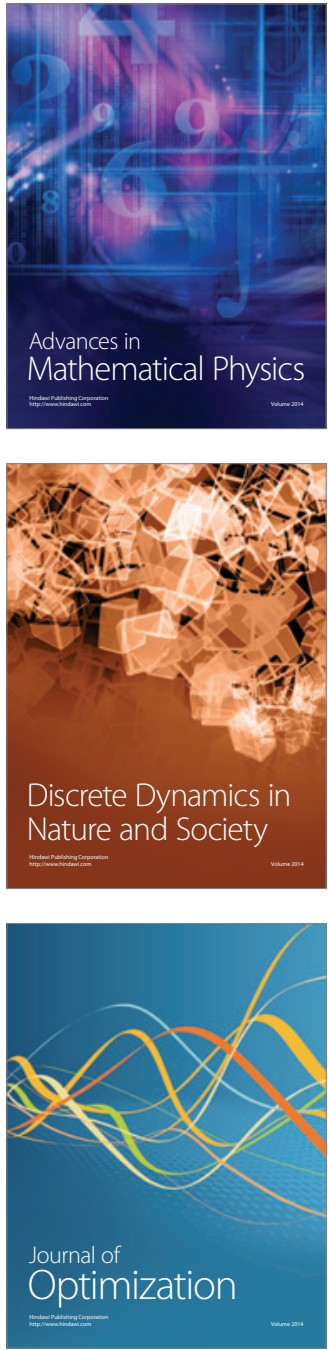